\title{
MEH-PPV film thickness influenced fluorescent quenching of tip-coated plastic optical fiber sensors
}

\begin{abstract}
The performance of plastic optical fiber sensors in detecting nitro aromatic explosives 1,4dinitrobenzene (DNB) have been investigated by fluorescence spectroscopy and analyzed by using fluorescence quenching technique. The plastic optical fiber utilized is 90 degrees cut tip and dip-coated with conjugated polymer MEH-PPV poly[2-methoxy-5-(2-ethylhexyloxy)1,4-phenylenevinylene] thin films for detection conjugants. The thicknesses of the MEH-PPV coating were varied to improvise the sensitivity whilst slowly reducing the fluorescence intensity. It was shown that fluorescence intensity from thinner film decreased by $(82 \%$ in $40 \mathrm{~s}$ ) in the presence of DNB signifying an improvement of $28 \%$ reduction with time $13 \mathrm{~s}$ less than that of the thicker film.
\end{abstract}

Keyword: Plastic optical fiber tip; Film thickness; Explosive; Fluorescence quenching 